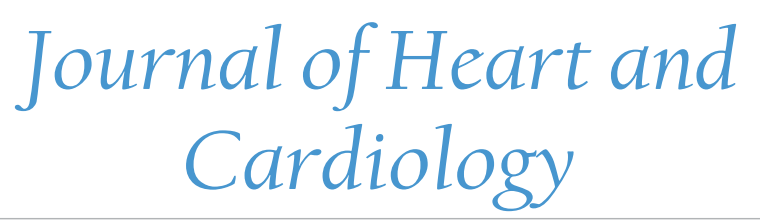

\title{
Effect of Tai Chi on Vascular Function among Patients with Peripheral Neuropathy
}

\author{
Arturo A. Arce-Esquivel ${ }^{1 *}$, Joyce E. Ballard ${ }^{1}$, Barbara K. Haas ${ }^{2}$, Melinda L. Hermanns², Carol A. \\ Rizer $^{2}$, Gary T. Kimmel ${ }^{3}$, and Yong T. Wang ${ }^{2}$
}

${ }^{1}$ Department of Health and Kinesiology, College of Nursing and Health Sciences, The University of Texas at Tyler, Tyler, Texas ${ }^{2}$ School of Nursing, The University of Texas at Tyler, Tyler, Texas

${ }^{3}$ Cancer Foundation for Life, Tyler, Texas

*Corresponding author: Arturo A. Arce-Esquivel, M.D., Ph.D., Department of Health and Kinesiology, College of Nursing and Health Sciences, The University of Texas at Tyler, 3900 University Blvd, Tyler, TX 75799, Tel: (903) 565-5838; Fax: (903) 566-7065; E-mail: aarce@uttyler.edu

\begin{abstract}
Foot pain due to Peripheral Neuropathy $(\mathrm{PN})$ is one of the factors affecting walking ability. It has been reported that diminished vascular function contributes to a decrease in physical function in individuals with PN. Microvascular disturbances have been reported in humans with neuropathic pain. Tai Chi, a Chinese conditioning exercise, has been associated with enhanced endothelial function. However, the effect of Tai Chi training on microvascular function in patients with PN has not been studied. This study aimed to assess the effects of Tai Chi on vascular function (i.e., vascular reactivity) and functional exercise capacity among patients with PN. Thirty-seven participants $(\operatorname{men}=21$, women $=16)$ were randomly assigned to either Tai Chi exercise (Ex, $n=20$, age: $71 \pm 9.50$ years) or control group (Con, $n=17$, age: $75 \pm 9.02$ years). Exercise training consisted of 12-week progressive Tai Chi (i.e., Yang Style), offered 3 times per week, 60 minutes sessions. The Con group did not participate in any exercise activity. Before and after training, vascular function [finger tip digital thermal monitoring of vascular reactivity] and functional exercise capacity [Six-Minute Walk test $(6 \mathrm{MW})]$ were evaluated. The Ex group experienced a significant $25 \%$ increase in vascular reactivity index from baseline $[1.93 \pm 0.43$ to $2.41 \pm 0.47,(P<0.05)]$. In addition, the $6 \mathrm{MW}$ test increased significantly in the Ex group by $28 \%$ [pre $=392 \pm$ 93; post $=503 \pm 105 \mathrm{~m},(P<0.05)]$. In the control group no significant changes were observed in either vascular function $[1.83 \pm 0.43$ to $1.81 \pm 0.37]$ or in the $6 \mathrm{MW}$ test $[393 \pm 142$ to $398 \pm 149 \mathrm{~m}]$. Participants experienced no complications and/or falls as a result of the intervention. These findings demonstrated that in patients with PN, a 12-week progressive Tai Chi exercise program was capable of increasing not only vascular function, specifically vascular reactivity index, but also of increasing the distance covered during the $6 \mathrm{MW}$ test. Clearly, this study underlies the importance of Tai Chi as an effective and safe exercise intervention suitable for patients with PN.
\end{abstract}

Received Date: August 03, 2016

Accepted Date: October 262016

Published Date: November 03, 2016

Citation: Arce-Esquivel, A.A., et al. Effect of Tai Chi on Vascular Function among Patients with Peripheral Neuropathy. (2016) J Heart Cardiol 2(2): 80- 86.

DOI: $10.15436 / 2378-6914.16 .1039$

Keywords: Microvascular function; Functional capacity; Neuropathy; Exercise

\section{Introduction}

Peripheral Neuropathy $(\mathrm{PN})$ is one of the most common disorders seen in general neurology and neuromuscular specialty clinics $^{[1]}$. Foot numbness and pain due to $\mathrm{PN}$ is one of the factors affecting walking ability. As a result, a large number of individuals 
with PN must cope with the consequences of reduced physical activity. An estimated 20 million people in the United States suffer from PN, a pathology associated with damage to peripheral nerves that results in loss of function, amputation, and decreased quality of life ${ }^{[2]}$. The prevalence of PN increases significantly in older adults and nearly one-third of all diabetics suffer from this condition ${ }^{[3]}$. Indeed, the Neuropathy Association ${ }^{[4]}$ estimates that there are currently 15 - 18 million Americans with Diabetic PN (DPN) due to the increasing prevalence of diabetes. The growing DPN statistic significantly raises the overall number of people with neuropathy in the U.S. Recent projections estimate that by 2050 , as many as one in every three Americans will have Type 2 Diabetes Mellitus (T2DM) ${ }^{[5]}$.

PN has numerous causes, including metabolic, infectious, toxic, inflammatory, ischemic, paraneoplastic and inherited disorders. Among patients with PN with a definable cause, T2DM is the most common cause worldwide ${ }^{[6]}$. However, twothirds of PN cases remain undiagnosed, relegated to the nebulous designation of "idiopathic" or "cryptogenic" neuropathy ${ }^{[1,7]}$. Irrespective of etiology, pathological changes associated with the disease are in many cases consistent with ischemia, secondary to vascular insufficiencies ${ }^{[8]}$.

The endothelium, a thin single sheet of endothelial cells, is a metabolically active layer that coats the inner surface of blood vessels and acts as an interface between the circulating blood and the vessel wall. The endothelium through the secretion of vasodilators, including nitric oxide (NO), and vasoconstrictors serves as a critical mediator of vascular homeostasis. A shift in the functions of the endothelium towards vasoconstriction, pro-inflammatory and pro-thrombotic states characterize improper functioning of these cells, leading to endothelial dysfunction (ED), implicated in the pathogenesis of many diseases including diabetes ${ }^{[9]}$.

The literature has clearly established that chronic ischemia contributes to demyelination or axonal degeneration ${ }^{[10]}$. Furthermore, DPN is partly a consequence of diabetes-mediated impairment in blood flow to, and resultant hypoxia of, nerves ${ }^{[1]}$. These neurological changes give rise to a variety of symptoms including reduced or altered sensation, pain, muscle weakness, and chronic fatigue. For instance, patients with Peripheral Arterial Disease (PAD) reportedly have a slower nerve conduction velocity in their legs, which is considered to be a clinical marker of PN, than individuals without $\mathrm{PAD}^{[12]}$. This clearly suggests a link between vascular deficiencies (or ED) and neural function in those patients ${ }^{[2]}$. Indeed, reduction of nerve conduction velocity in DPN has been shown to be preceded by impaired vasodilation of the arterioles ${ }^{[13]}$. Clearly, these findings suggest that signs of microvascular disease are present prior to the development of clinically overt DPN. Finally, it has been reported that diminished vascular function contributes to a decrease in physical function in individuals with PN, compromising arterial vasculature $^{[14]}$.

Importantly, DPN introduces adverse changes at all levels of the vascular tree, electing both macrovascular and microvascular complications such as increased arterial stiffness ${ }^{[15]}$. It is estimated that the microvasculature (small, resistance or pre-capillary vessels) with an internal diameter of less than 100 microns) accounts for over $95 \%$ of the total body vasculature. Such a compromised macro and micro vascular system could certainly contribute to the progression of the disease. In fact, the literature provides significant evidence that conditions of acute and chronic ischemia contribute to changes in peripheral nerve function and structure ${ }^{[10]}$.

Regular exercise training (e.g., aerobic and resistance training) has shown to improve vascular function in T2DM patients ${ }^{[16]}$. Therefore, it would be reasonable to speculate that physical training may be an excellent way to preserve vascular function and functional exercise capacity among patients with PN. However, the body of research related to exercise and PN is inconclusive. A systematic review of exercise for persons with $\mathrm{PN}$, regardless of etiology, concluded there is inadequate evidence to evaluate the effects of exercise on physical exercise ability in these patients ${ }^{[17]}$. Tai Chi, a mind-body harmony exercise, is an ancient Chinese healing/martial art that consists of a series of graceful movements with deep and slow diaphragmatic breathing. Substantial evidence exists on the cardiorespiratory fitness and functional capacity benefits of Tai Chi in older patients with chronic disabilities ${ }^{[18-20]}$. It has been reported that regular practice of Tai Chi is associated with enhanced endothelium-dependent dilation in skin vasculature of healthy older individuals ${ }^{[21]}$. Furthermore, healthy elder Tai Chi practitioners had higher cutaneous microcirculatory function during exercise than did their sedentary counterparts ${ }^{[22]}$. We are unaware of any study that has examined the effect of Tai Chi exercise training on peripheral microcirculation. Thus, the purpose of this study aimed to assess the effects of progressive Tai Chi exercise training on microvascular function and functional exercise capacity among patients with $\mathrm{PN}$.

\section{Methods}

\section{Study participants}

Thirty-seven participants $(\mathrm{men}=21$, women $=16)$ with a physician's diagnosis of PN were recruited from the East Texas area to participate in this study. The inclusion criteria for the study were (a) ability to maintain an upright posture for at least one minute voluntarily; (b) ability to walk at least 20 yards independently; (c) willingness to participate in the study; and (d) a diagnosis of bilateral PN. Individuals were excluded from participation if there was (a) a history or evidence of central nervous system dysfunction; (b) musculoskeletal deformity which could influence gait and balance; (c) a history or evidence of vestibular dysfunction; (d) a history of angina; (e) evidence of plantar ulcer; and (f) unstable disease ${ }^{[1]}$ (e.g. uncontrolled diabetes mellitus, arthritis, coronary artery disease, etc.). Following explanation of all the details of the study, each participant signed an informed consent approved by the Institutional Review Board.

\section{Experimental design}

The thirty-seven participants were randomly assigned to either Tai Chi exercise (Ex, $n=20)$ or control group (Con, $n$ = 17). The Health and Physical Activity Questionnaire ${ }^{[23]}$ was used to determine the level of physical activity behavior, and the medical history of each participant before the study started. Exercise training consisted of 12-week progressive Tai Chi (i.e., Yang Style), offered 3 times per week, 60 minutes sessions. The Con group did not participate in any exercise activity during the

${ }^{1}$ Unstable disease (i.e., change in medication within the last 6 months). 
study. They just kept doing their daily routines. The proposed study was a randomized controlled design to determine the effectiveness of a 12-week organized progressive Tai Chi exercise program. Each participant in the Ex and Con groups was examined during two separate visits (i.e., before and after the 12-week exercise program) for the measurement of both vascular function and functional exercise capacity.

\section{Vascular function}

The fingertip Digital Thermal Monitoring (DTM), a non-invasive test, of vascular reactivity (i.e., microvascular function) was used ${ }^{[24]}$. All DTM measurements were performed in a quiet, dimly lit room with a controlled ambient temperature between 23 and $25^{\circ} \mathrm{C}$. The studies were conducted after an overnight fast of at least 10 hours (water was permitted) and abstinence from tobacco, alcohol, caffeine, vasoactive medications, exercise, high-fat foods, and vitamin C. The measurements were obtained with the subjects seated for 20 minutes of rest. Segmental pressure cuffs were positioned around the forearms. Disposable finger probes were attached to the index fingers of both hands. DTM of both hands was obtained during 5-minute stabilization, 5-minute cuff inflation, and 5-minute deflation using an automated, operator independent protocol (VENDYS, Endothelix Inc., Houston, TX, USA). The right upper arm cuff (i.e., occluded arm) was rapidly inflated to $50 \mathrm{mmHg}$ above systolic pressure for 5 minutes and then rapidly deflated to invoke reactive hyperemia distally. Thermal changes during a 5-minute arm-cuff-induced reactive hyperemia test were monitored continuously in the fingertip of both the occluded (i.e., right) and the non-occluded (i.e., left) arms using VENDYS software. Finally, the area under the temperature curve was used to determine the vascular reactivity index (VRI) which assessed the difference in response in microvascular function between the two groups.

The DTM technology has been directly shown to be a valuable indicator of overall cardiovascular risk ${ }^{[25,26]}$. Previous studies have demonstrated the ability of the DTM test to discriminate individuals with established Cardiovascular Disease (CVD) or high risk of future CVD in normal and low-risk individuals ${ }^{[27,28]}$. The repeatability of DTM is excellent and can be used as a reproducible and operator-independent test for non-invasive measurement of vascular function ${ }^{[2,29,30]}$. Indeed, DTM of vascular reactivity appears to be an appropriate test to evaluate vascular reactivity in clinical and research settings ${ }^{[2,229]}$.

\section{Functional exercise capacity}

A Six-Minute Walk (6MW) test was administered using a standardized protocol outlined by the American Thoracic Society ${ }^{[31]}$. Briefly, participants were instructed to walk at their own pace trying to cover as much distance as possible in $6 \mathrm{~min}-$ utes on an indoor track. In order to standardize the protocol, the participants were not coached during the test, but made aware of time remaining to completion. The distance covered was recorded to the nearest meter. Maximum walking distance traveled in 6 minutes, to the nearest meter, was recorded and used for analysis.

The 6MW test is a valid measure of "functional exercise capacity" as it evaluates the integrated response of all systems involved during physical activity ${ }^{[31]}$. It is therefore frequently used in both elderly and diseased populations, and was the rationale for using it in the current study.

\section{Exercise program}

Exercise consisted of 12-week progressive Tai Chi (i.e., Yang Style), offered 3 times per week, 60 minutes each time. The Yang style is the most popular form of Tai $\mathrm{Chi}^{[32]}$. A typical Tai Chi practice session lasted 60 minutes included diaphragmatic breathing and gentle stretching exercises for warm-up (10 min), learning and practicing Tai Chi Yang Style 10 forms (45 $\mathrm{min}$ ), and cool down (5 min). During the Tai Chi training sessions, participants were led by a Tai Chi Master and imitated the motions and postures at the same speed. In addition, other well trained individuals were present to observe and to ensure safety among the participants.

\section{Statistical analysis}

All values are means \pm Standard Deviation (SD) or \pm Standard Error (SE) when appropriate. Analyses were performed using paired samples t-tests (two-tailed), Chi square, and/or ANOVA for repeated measurements as appropriate. All statistical analyses were performed with Graph Pad Prism version 6.0a (Graph Pad Software, Inc. CA, USA). The level of significance was set at $P<0.05$.

\section{Results}

\section{Participant characteristics}

A total of 37 individuals with bilateral PN participated in all aspects of this study. Participants were assigned to Ex ( $n=21$; age: $71 \pm 9.50$ years) or Con $(n=16$; age: $75 \pm 9.02$ years) groups. The participant characteristics are presented in Table 1. Forty-three percent of the participants were women, and $57 \%$ men. The oldest participant tested was 90 years old, and the youngest was 50 years old. Eighty four percent of the participants were over the age of 65 . The physical activity behavior indicated that these participants were low-to-moderately active. The length of time that subjects held the diagnosis of PN ranged from 12 months to 20 years, with $60 \%$ of the participants experiencing PN for over 10 years. The etiology of PN was T2DM in approximately $30 \%$ of the subjects. In $8 \%$ of patients the etiology was chronic low back pain, and $3 \%$ of patients were cancer survivors. In the remaining subjects (59\%) the etiology was unknown. Forty-three percent of the patients had stage I hypertension (i.e., physician diagnosed) on the basis of resting systolic blood pressure ( $\mathrm{SBP} \geq 140 \mathrm{mmHg}$ ). There was no significant difference between the Con and Ex in age, body mass index, systolic and diastolic blood pressures, or heart rate.

\section{Vascular function}

The average values for the vascular function assessments between the Con and Ex groups are presented in Table 2 and Figure 1. The average baseline VRI was $1.83 \pm 0.43$ and $1.93 \pm 0.43$ for the Con and Ex groups, respectively. Following the 12-week Tai Chi exercise program, the VRI (i.e., microvascular function) increased significantly in the Ex group. Indeed, the Ex group experienced a 25\% increase from baseline (1.93 \pm 0.43 to $2.41 \pm 0.47, P<0.05)$. Finally, the post-intervention VRI for the Con group remained almost the same as baseline (1.81 \pm $0.37)$. 
Table 1: Participant Characteristics.

\begin{tabular}{|l|c|c|}
\hline Variable & Con $(\mathbf{n}=\mathbf{1 7})$ & Ex $(\mathbf{n}=\mathbf{2 0})$ \\
\hline Age $(\mathrm{y})$ & $75 \pm 9.02$ & $71 \pm 9.50$ \\
\hline Height $(\mathrm{cm})$ & $170 \pm 12.04$ & $173 \pm 8.63$ \\
\hline Weight $(\mathrm{kg})$ & $83 \pm 20.15$ & $87 \pm 12.92$ \\
\hline BMI $\left(\mathrm{kg} \cdot \mathrm{m}^{-2}\right)$ & $28 \pm 5.69$ & $29 \pm 3.82$ \\
\hline Resting SBP $(\mathrm{mmHg})$ & $142 \pm 18$ & $137 \pm 14$ \\
\hline Resting DBP $(\mathrm{mmHg})$ & $75 \pm 11$ & $76 \pm 7$ \\
\hline Resting HR $(\mathrm{bpm})$ & $66 \pm 10$ & $67 \pm 10$ \\
\hline
\end{tabular}

Values are means $\pm \mathrm{SD}$. y: years; cm: centimeters; kg: kilograms; BMI: Body Mass Index; SBP: Systolic Blood Pressure; mmHg, DBP: Diastolic Blood Pressure; mmHg, HR: Heart Rate; bpm: beats per minute.

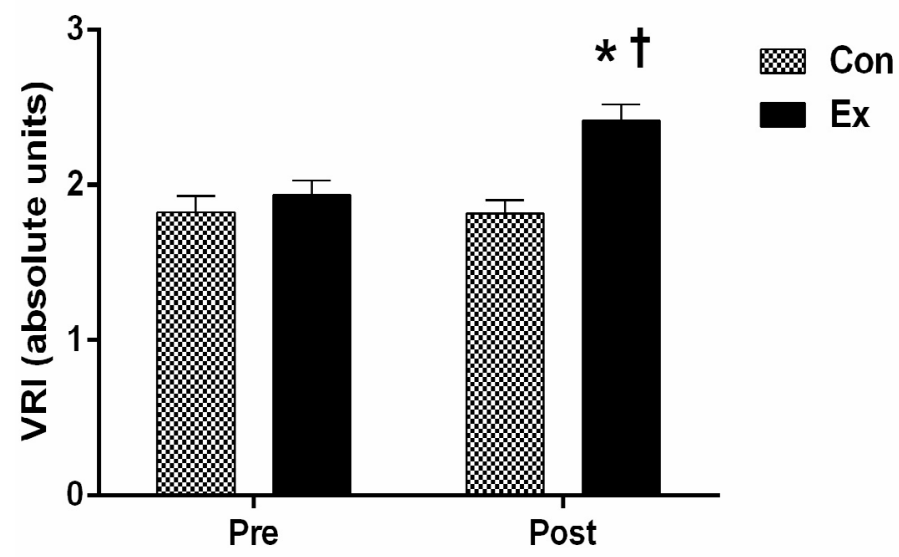

Figure 1: Changes in Vascular Reactivity Index (VRI) between the Con and Ex groups. Values are means \pm S.E. $* P<0.05$ for Con-pre and Con-post vs. Ex-post; $\uparrow P<0.05$ for Ex-pre vs. Ex-post.

\section{Physical exercise capacity}

The average values for the physical exercise capacity between the Con and Ex groups are presented in Table 2 and Figure 2. The average baseline 6MW test was $393 \pm 142$ and $392 \pm 93 \mathrm{~m}$ for the Con and Ex groups, respectively. Following the 12-week Tai Chi exercise program, the distance covered in the $6 \mathrm{MW}$ increased significantly in the Ex group. Indeed, the Ex group experienced a $28 \%$ increase from baseline (392 \pm 93 to $503 \pm 105 \mathrm{~m}, P<0.05)$. Finally, the post-intervention $6 \mathrm{MW}$ test for the Con group remained almost the same as baseline (398 \pm $149 \mathrm{~m})$.

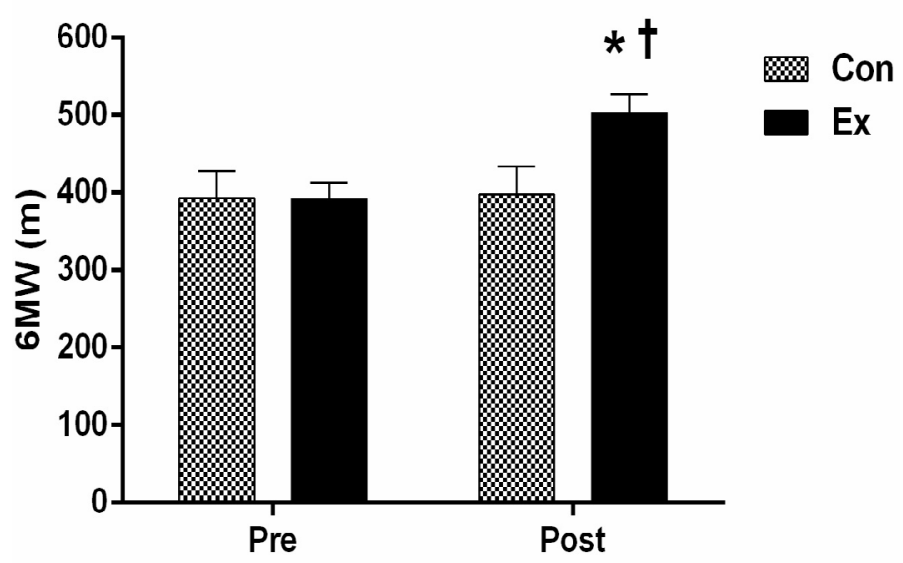

Figure 2: Six-Minute Walk Test $(6 \mathrm{MW})$ between the Con and Ex groups. Values are means \pm S.E. $* P<0.05$ for Con-pre and Con-post vs. Ex-post; $\uparrow P<0.05$ for Ex-pre vs. Ex-post.

Table 2: Vascular and Functional Assessments.

\begin{tabular}{|l|c|c|c|c|}
\hline Variable & $\begin{array}{c}\text { Con }(\mathbf{n}=\mathbf{1 7}) \\
\text { Pre }\end{array}$ & $\begin{array}{c}\text { Con }(\mathbf{n}=\mathbf{1 7}) \\
\text { Post }\end{array}$ & $\begin{array}{c}\text { Ex (n= 20) } \\
\text { Pre }\end{array}$ & $\begin{array}{c}\text { Ex (n= 20) } \\
\text { Post }\end{array}$ \\
\hline Vascular Function & $1.83 \pm 0.43$ & $1.81 \pm 0.37$ & $1.93 \pm 0.43$ & $2.41 \pm 0.47 * \dagger$ \\
\hline VRI (absolute units) & $393 \pm 142$ & $398 \pm 149$ & $392 \pm 93$ & $503 \pm 105 * \dagger$ \\
\hline Functional Exercise Capacity &
\end{tabular}

Values are means \pm SE. VRI: Vascular Reactivity Index; 6 MW: 6-Minute Walk Test; m: meters.

$* P<0.05$ for Con-pre and Con-post vs. Ex-post; $\uparrow P<0.05$ for Ex-pre vs. Ex-post comparison at the measured characteristic.

\section{Discussion}

The present data indicated that in patients with $\mathrm{PN}$, a 12-week progressive Tai Chi program is capable of increasing not only vascular function (i.e., microvascular function), but also increasing the distance covered during the $6 \mathrm{MW}$ test. Clearly, this study underlies the importance of Tai Chi as an effective and safe exercise intervention suitable for patients with PN.

\section{Vascular function}

The DTM is a non-invasive technique to study vascular reactivity (i.e., microvascular function) in humans. Briefly, a standard arm-cuff promotes a temporary occlusion of blood flow in the arm. During the cuff occlusion, the lack of blood flow (i.e., ischemia) elicits a microvascular dilative response. Upon releasing the cuff, blood flow rushes into the forearm and hand, not only restoring baseline flow but also resulting in an overshoot (i.e., reactive hyperemia). The reactive hyperemia promotes shear stress in the larger arteries to dilate and accommodate the increased blood flow. In general, the study of blood flow responses following occlusion indicates that younger, fitter, and healthier individuals exhibit greater flow responses, suggesting better vascular function. Reductions in the hyperemic response, a hallmark feature of impaired vascular function, are observed during DTM. Indeed, the VRI provides a non-invasive 
"window" to the cardiovascular system allowing the detection, measurement, and monitoring of vital patient information such as overall cardiovascular health. The VRI is reported in absolute units using a vascular reactivity scale: a) $<1$ ("poor"), b) 1 - 2 ("intermediate"), and c) > 2 ("good") (VENDYS, Endothelix Inc., Houston, TX, USA).

The average baseline VRI was $1.83 \pm 0.43$ and 1.93 \pm 0.43 for the Con and Ex groups, respectively (Table 2, Figure 1). These vascular assessments placed the participants in the "intermediate" category of vascular reactivity $(1-2)$ at the beginning of the intervention. Together, these findings suggest that microvascular function in individuals with $\mathrm{PN}$ is compromised. Such a compromised vascular function could certainly contribute to the progression of the disease. In fact, the literature provides significant evidence that conditions of acute and chronic ischemia contribute to changes in peripheral nerve function and structure $^{[10]}$. Laghi Pasini et al. ${ }^{[10]}$, reported a strong correlation between severity of nerve damage and stage of vascular insufficiency. It is not currently known what underlying mechanisms may contribute to deterioration of peripheral nerve function, but an increase in intracellular calcium ${ }^{[33]}$, and/or reactive oxygen species (free radicals) ${ }^{[34]}$ could disrupt myelin and lead to axonal fiber degeneration.

On the other hand, it is well-known that exercise training is able to improve vascular function in diseased individuals. For instance, regular exercise training improved vascular function in T2DM patients ${ }^{[16]}$. Indeed, forearm blood flow (FBF) response to Acetylcholine and flow-mediated dilation (or FMD) of the brachial artery improved significantly after an 8-week exercise program (combined aerobic and resistance training) in T2DM patients ${ }^{[35]}$. In another study, a short-term exercise program ( 8 weeks) improved brachial FMD in patients at risk for and with coronary artery disease ${ }^{[36]}$. Similarly, a combined aerobic and resistance exercise training program improved FBF in patients with chronic heart failure ${ }^{[37]}$. The improvements in vascular function reported in those studies appeared to be NO-mediated.

There is evidence that Tai Chi promotes beneficial effects (e.g., improved cardiorespiratory fitness and functional capacity) among older patients with chronic disabilities ${ }^{[18-20]}$. In addition, it has been reported that Tai Chi could also improve vascular function among healthy individuals ${ }^{[21,22]}$. For instance, regular practice of Tai Chi is associated with enhanced endothelium-dependent dilation in skin vasculature of healthy older individuals when compared to sedentary men ${ }^{[21]}$. These investigators suggested that Tai Chi might be able to enhance NO/ endothelium derived relaxing factor bioactivity in other parts of the circulation in older men. Another study aimed to examine the effect of Tai Chi training on cutaneous microcirculation and the release of NO induced by acute exercise in healthy elderly individuals $^{[22]}$. These findings indicated that elder Tai Chi practitioners had higher cutaneous microcirculatory function during exercise (i.e., bicycle maximal graded exercise test) than did their sedentary counterparts ${ }^{[22]}$. This change may be partially mediated by enhancement of NO release. Indeed, the Tai Chi group had higher levels of plasma nitrite and nitrate than the sedentary group at rest and immediately after exercise ${ }^{[22]}$.

Importantly, our findings reported that following a 12week progressive Tai Chi exercise program, the VRI increased significantly in the Ex group. Truly, the Ex group experienced a $25 \%$ increase from baseline $(1.93 \pm 0.43$ to $2.41 \pm 0.47, P<$ 0.05 ) (Table 2, Figure 1). To our knowledge, this is the first study that has shown Tai Chi exercise training was able to improve peripheral microcirculation among patients with PN. We speculated that the increased microvascular reactivity, observed in our study, was mediated also by enhancement of NO release as reported previously.

\section{Physical exercise capacity}

A decline in physical functional performance in individuals with $\mathrm{PN}$ is widely accepted, although the rate of decline and contributing mechanisms are much less understood. The $6 \mathrm{MW}$ test is an easily implemented test that determines the maximum distance a person can walk in 6-min. The baseline group averages for the 6MW in this study (Con: $392 \pm 93$ m; Ex: $393 \pm$ $142 \mathrm{~m}$ ) (shown in Table 2 and Figure 2) appears to be quite typical for those seen in patients with heart failure $(396 \pm 92 \mathrm{~m})^{[38]}$, indicating significant functional limitations. Importantly, based on the literature indicating that a walking distance less than 400 $\mathrm{m}$ is associated with higher prevalence of institutionalization, morbidity, mortality, hospitalization and transplantation ${ }^{[39]}$, the present baseline data suggests that $54 \%$ of the patients with PN are at an increased risk of complications.

We reported here that following the 12-week Tai Chi exercise program, the distance covered in the $6 \mathrm{MW}$ increased significantly in the Ex group. Truly, the Ex group experienced a $28 \%$ increase from baseline $(392 \pm 93$ to $503 \pm 105 \mathrm{~m}, P<$ 0.05 ) (Table 2, Figure 2). It is important to notice the potential association between vascular responses and the 6MW test. Indeed, the vascular reactivity assessments reported in this study provide evidence of the ability of the vasculature to respond to an increase in metabolic demand (i.e., 5 minutes of occlusion). Arguably, the physiologic demands associated with the walking test would also serve to challenge the vasculature, and consequently individuals who have evidence of ED would have reduced physical exercise capacity.

We remain cautious in our interpretations. The lack of a mechanistic approach in the current study prevents more sophisticated speculation regarding the underlying positive effects of Tai Chi on microvascular function and physical exercise capacity. However, these findings are particularly interesting since the existing evidence clearly indicates that lower cardiorespiratory fitness is a significant predictor of dependence ${ }^{[40]}$ and that Ankle-Brachial Index test (ABI) is a strong predictor of the degree of physical decline in patients with $\mathrm{PAD}^{[41]}$. So, it is our hope that these data would stimulate clinicians to consider assessing vascular function in patients with $\mathrm{PN}$, in an effort to better understand patients' physical exercise capacity impairment.

\section{Conclusions}

In conclusion, the present findings demonstrated that in patients with PN, a 12-week progressive Tai Chi exercise program is capable of increasing not only microvascular function, but also of increasing the distance covered during the 6MW test. Considering the gait and balance challenges associated with PN, low velocity, low impact exercises, like Tai Chi, might be the most effective form of exercise for this clinical population. The present study underlies the importance of Tai Chi as an effective and safe exercise intervention suitable for patients with PN 
because of its slow and structured movements. Finally, Tai Chi is a low-technology approach to conditioning that can be implemented in the community with minimal cost.

Acknowledgement: The authors gratefully acknowledge Linda Friedemann, Sowmya Yarlagadda (graduate student research assistant), Crystal Bryce, Laura Hoyt, and Kleanthe Caruso for their technical assistance.

Grants: This research was supported by The University of Texas at Tyler - College of Nursing and Health Sciences Intramural Grant Program (2015 - Arturo A. Arce-Esquivel).

Conflicts of Interest: No conflicts of interest, financial or otherwise, are declared by the authors.

\section{References}

1. Gordon Smith, A., Robinson Singleton, J. Idiopathic neuropathy, prediabetes and the metabolic syndrome. (2006)J Neurol Sci 242(1-2): 9-14.

2. National Institute of Neurological Disorders and Stroke. (2014) Peripheral Neuropathy Fact Sheet.

3. Departments of Labor, Health and Human Srevices, and Education, and Related Agencies Appropriation Bill, 2005. (2004) Congress 108.

4. Increase in Diabetic Neuropathy Escalates Neuropathy Epidemic Impacting Millions According to The Neuropathy Association. (2013) The Neuropathy Association.

5. Boyle, J.P., Thompson, T.J., Gregg, E.W., et al. Projection of the year 2050 burden of diabetes in the US adult population: dynamic modeling of incidence, mortality, and prediabetes prevalence. (2010) Popul Health metr 8: 29

6. Hughes, R.A. Peripheral neuropathy. (2002) BMJ 324(7335): 466469.

7. England, J.D., Asbury, A.K. Peripheral neuropathy. (2004) Lancet 363(9427): 2151-2161.

8. Krishnan ,A.V., Lin, C.S., Kiernan, M.C. Nerve excitability properties in lower-limb motor axons: evidence for a length-dependent gradient. (2004) Muscle Nerve 29(5): 645-655.

9. Widlansky, M.E., Gokce,N., Keaney, J.F., et al. The clinical implications of endothelial dysfunction. (2003) J Am Coll Cardiol 42(7): 1149-1160.

10. Laghi, Pasini, F., Pastorelli, M., Beermann, U., et al. Peripheral neuropathy associated with ischemic vascular disease of the lower limbs. (1996) Angiology 47(6): 569-577.

11. Tremont-Lukats, I.W., Megeff, C., Backonja, M.M. Anticonvulsants for neuropathic pain syndromes: mechanisms of action and place in therapy. (2000) Drugs 60(5): 1029-1052.

12. Regensteiner, J.G., Wolfel, E.E., Brass, E.P., et al. Chronic changes in skeletal muscle histology and function in peripheral arterial disease. (1993) Circulation 87(2): 413-421.

13. Coppey, L.J., Davidson, E.P., Dunlap, J.A., et al. Slowing of motor nerve conduction velocity in streptozotocin-induced diabetic rats is preceded by impaired vasodilation in arterioles that overlie the sciatic nerve. (2000) Int J Exp Diabetes Res 1(2): 131-143.

14. Arce-Esquivel, A.A., Manor, B., Li, L., et al. Relationship Between Vascular and Physical Function in Individuals with Peripheral Neuropathy. (2009) Vascular Disease Prevention 6: 148-156.

15. Kozakova, M., Morizzo, C., Bianchi, C., et al. Glucose-related arterial stiffness and carotid artery remodeling: a study in normal subjects and type 2 diabetes patients. (2014) J Clin Endocrinol metab 99(11): E2362-2366.

16. Maiorana, A., O'Driscoll, G., Cheetham, C., et al. The effect of combined aerobic and resistance exercise training on vascular function in type 2 diabetes. (2001) J Am Coll Cardiol 38(3): 860-866.
17. White, C.M., Pritchard, J., Turner-Stokes, L. Exercise for people with peripheral neuropathy. (2004) Cochrane Database Sys Rev (4): CD003904.

18. Song, R., Lee, E.O., Lam, P., et al. Effects of tai chi exercise on pain, balance, muscle strength, and perceived difficulties in physical functioning in older women with osteoarthritis: a randomized clinical trial. (2003) J Rheumatology 30(9): 2039-2044.

19. Ades, P.A., Wu, G. Benefits of tai chi in chronic heart failure: body or mind? (2004) American J med 117(8): 611-612.

20. Wang, C., Collet, J.P., Lau, J. The effect of Tai Chi on health outcomes in patients with chronic conditions: a systematic review. (2004) Arch intern med 164(5): 493-501.

21. Wang, J.S., Lan. C., Chen, S.Y., et al. Tai Chi Chuan training is associated with enhanced endothelium-dependent dilation in skin vasculature of healthy older men. (2002) J Am Geriatr Soc 50(6): 1024-1030. 22. Wang, J.S., Lan, C., Wong, M.K. Tai Chi Chuan training to enhance microcirculatory function in healthy elderly men. (2001) Arch phy med rehabil 82(9): 1176-1180.

23. Jones, C.J., Rose, D. Physical Activity Instruction of Older Adults. (2005) Human Kinetics p: 69-73.

24. Ahmadi, N., McQuilkin, G.L., Akhtar, M.W., et al. Reproducibility and variability of digital thermal monitoring of vascular reactivity. (2011) Clin phys funct imaging 31(6): 422-428.

25. Dhindsa, M., Sommerlad, S.M., DeVan, A.E., et al. Interrelationships among noninvasive measures of postischemic macro- and microvascular reactivity. (2008) J Appl Physiol (1985) 105(2): 427-432.

26. Ahmadi, N., Tirunagaram, S., Hajsadeghi, F., et al. Concomitant insulin resistance and impaired vascular function is associated with increased coronary artery calcification. (2010) Int J cardiol 144(1): 163165.

27. Ahmadi, N., Hajsadeghi, F., Gul, K., et al. Relations between digital thermal monitoring of vascular function, the Framingham risk score, and coronary artery calcium score. (2008) J cardiovasc comput tomogr 2(6): $382-328$.

28. Ahmadi, N., Nabavi, V., Nuguri, V., et al. Low fingertip temperature rebound measured by digital thermal monitoring strongly correlates with the presence and extent of coronary artery disease diagnosed by 64-slice multi-detector computed tomography. (2009) Int J Cardiovasc Imaging 25(7): 725-738.

29. Zeb, I., Ahmadi, N., Molnar, M.Z., et al. Association of coronary artery calcium score and vascular dysfunction in long-term hemodialysis patients. (2013) Hemodial int Symposium on Home Hemodialysis 17(2): 216-222.

30. McQuilkin, G.L., Panthagani, D., Metcalfe, R.W., et al. Digital thermal monitoring (DTM) of vascular reactivity closely correlates with Doppler flow velocity. (2009) Conf Proc IEEE Eng Med Biol Soc 2009: 1100-1103.

31. ATS statement: guidelines for the six-minute walk test. (2002) Am J Respir Crit Care Med 166(1): 111-117.

32. Thomas, G.N., Hong, A.W., Tomlinson, B., et al. Effects of Tai Chi and resistance training on cardiovascular risk factors in elderly Chinese subjects: a 12-month longitudinal, randomized, controlled intervention study. (2005) Clin Endocrinol (Oxf) 63(6): 663-669.

33. Cheung, J.Y., Bonventre, J.V., Malis, C.D., et al. Calcium and ischemic injury. (1986) N Engl J med 314(26): 1670-1676.

34. Nagamatsu, M., Schmelzer, J.D., Zollman, P.J., et al. Ischemic reperfusion causes lipid peroxidation and fiber degeneration. (1996) Muscle nerve 19(1): 37-47.

35. Maiorana, A., O’Driscoll, G., Goodman, C., et al. Combined aerobic and resistance exercise improves glycemic control and fitness in type 2 diabetes. (2002) Diabetes Res clin pract 56(2): 115-123.

36. Green, D.J., Walsh, J.H., Maiorana, A., et al. Comparison of resistance and conduit vessel nitric oxide-mediated vascular function in vivo: effects of exercise training. (2004) J Appl Physiol 97(2): 749-755. 37. Maiorana, A., O'Driscoll, G., Dembo, L., et al. Effect of aerobic and resistance exercise training on vascular function in heart failure. (2000) Am J physio Heart Circ Physiol 279(4): H1999-2005. 
38. Opasich, C., Pinna, G.D., Mazza, A., et al. Six-minute walking performance in patients with moderate-to-severe heart failure; is it a useful indicator in clinical practice? (2001) Eur Heart J 22(6): 488-496.

39. Rolland, Y.M., Cesari, M., Miller, M.E., et al. Reliability of the 400$\mathrm{m}$ usual-pace walk test as an assessment of mobility limitation in older adults. (2004) J Am Geriatr Soc 52(6): 972-976.

40. Paterson, D.H., Govindasamy, D., Vidmar, M., et al. Longitudinal study of determinants of dependence in an elderly population. (2004) J Am Geriatr Soc 52(10): 1632-1638.

41. McDermott, M.M., Liu, K., Greenland, P., et al. Functional decline in peripheral arterial disease: associations with the ankle brachial index and leg symptoms. (2004) JAMA 292(4): 453-461.

Ommega Online Publishers

Journal Title: Journal of Heart and Cardiology

Short title : J Heart Cardiol
Journal ISSN: 2378-6914 (online)

Journal E-mail: cardiology@ommegaonline.com

Website: www.ommegaonline.org 